\title{
ROUTING IN THE AIRBORNE INTERNET
}

\author{
Daniel Medina, Felix Hoffmann, Francesco Rossetto \\ Institute of Communications and Navigation, German Aerospace Center (DLR), Munich, Germany \\ Carl-Herbert Rokitansky \\ Department of Computer Science, University of Salzburg, Austria
}

\begin{abstract}
The Airborne Internet is envisioned to be a large scale multihop wireless mesh network of civil aviation aircraft connected via long range highly directional air-to-air radio links. We propose a novel geographic load share routing metric to mitigate congestion in this network, taking into account the underlying link scheduling constraints with directional antennas. When forwarding packets for a given destination, a node considers not one but a set of next hop candidates, and spreads traffic among them based on queue dynamics. Our simulations show that introducing this flexibility in the routing function can greatly increase a node's ability to satisfy its bandwidth demands during link scheduling, yielding significant performance improvements in terms of network throughput and average packet delay. The ability to exploit this flexibility depends on the spatial reuse of the underlying network. For the simulated scenario, an increase in network throughput of $200 \%$ on average is shown, compared to a state-of-the-art geographic routing algorithm.
\end{abstract}

\section{Introduction}

Airborne mesh networks are self-organizing infrastructureless wireless networks formed by aircraft via direct air-to-air (A2A) radio links. Such networks have so far been considered mainly in the context of military aviation [1].

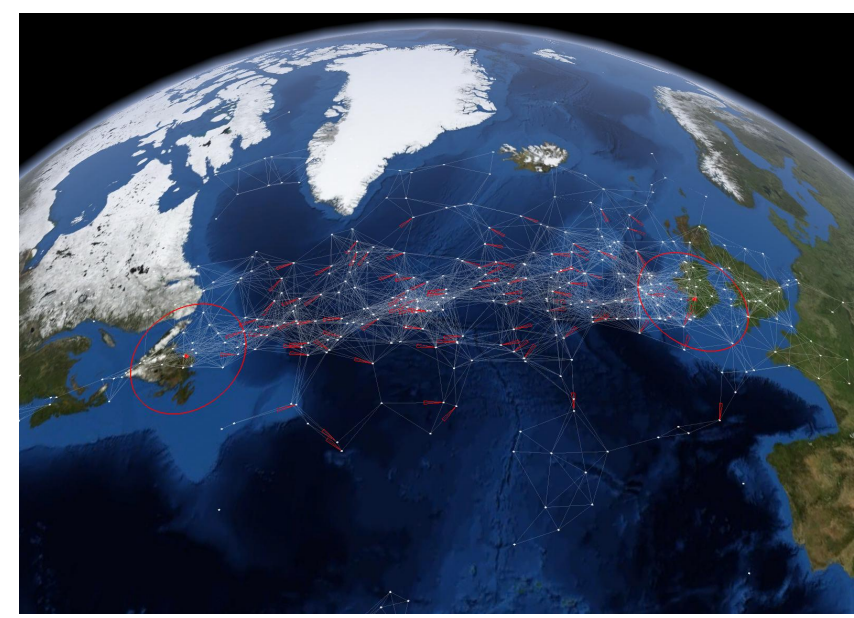

Figure 1 The Airborne Internet (North Atlantic Corridor). The North Atlantic is the busiest oceanic airspace in the world.

This paper considers the application of airborne mesh networking to civil aviation aircraft in what has recently been termed the "Airborne Internet" [2][3]. This technology can be used to extend the coverage of broadband air-to-ground (A2G) infrastructure networks, e.g., to provide inflight Internet access in regions where aircraft are unable to reach such infrastructure, such as over large bodies of water (Fig. 1). Nowadays, connectivity in such regions is only possible via satellite links, which are costly and suffer from long round trip times. With the Airborne Internet, airlines would forward each other's traffic in a cooperative way, resulting in lower communication costs, as well as reduced round trip times, enabling 
delay-sensitive applications, e.g., voice over IP (VoIP). Needless to say, for such a network to be feasible, there must be a critical mass of aircraft equipped with the appropriate technology.

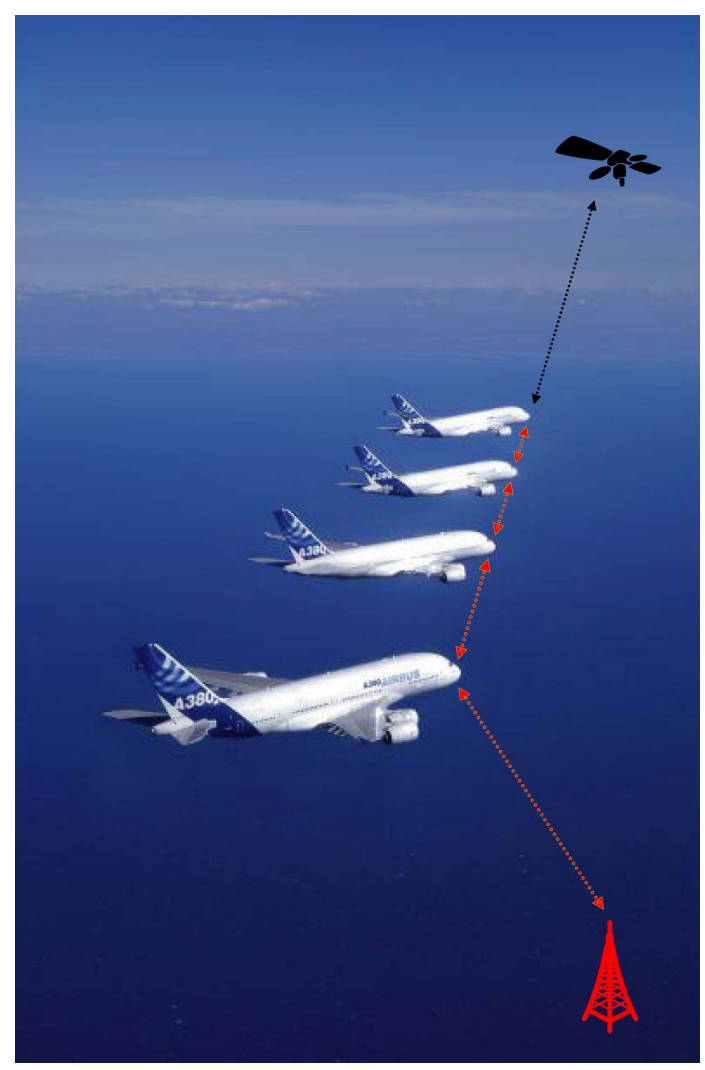

Figure 2 Airborne mesh networking.

Given the huge scale of the Airborne Internet compared to traditional mesh networks, the radio communication range must be large enough to guarantee network connectivity in regions and at times with low air traffic density. The line-of-sight (LOS) communication range between two aircraft is limited by the horizon (earth's curvature) and depends on the aircraft's flight level. At $35000 \mathrm{ft}$, air-to-air communication could be achieved in principle as far as $450 \mathrm{nmi}$ (nautical miles). At such distances, the use of highly directional antennas is crucial for broadband communication. Directional transmission may be realized using different technologies, such as digital beamforming or even optical communication links. The main disadvantage of optical links is their limited applicability in the presence of clouds, rain, etc. Aircraft usually fly above the clouds, but the line of sight will typically traverse a portion of the troposphere if long range communication is to be realized.

A key advantage of directional antennas is increased spatial reuse. By directing the beam toward the intended receiver, the energy radiated in other directions is minimal, and therefore the number of interfered nodes is reduced. If beamforming is also used in reception, the increase in spatial reuse is even higher, since the receiver rejects energy arriving from directions away from the intended transmitter. These techniques have been shown to tremendously increase the capacity of wireless networks [4][5].

Broadband air-to-air communication requires a medium access control (MAC) protocol capable of handling high traffic loads in the network and providing quality-of-service (QoS) guarantees to communicating nodes. Carrier sense multiple access (CSMA) techniques are inappropriate in this environment, since their performance degrades significantly as the traffic load and network size grow due to increased probability of collisions and the presence of hidden terminals. Aircraft are equipped with GPS for navigation purposes, and this provides a global time reference that can be exploited for channel access synchronization among network nodes, e.g., to schedule collision-free transmissions in a time division multiple access (TDMA) fashion.

The location information provided by GPS can be exploited for position-based forwarding of packets from source node to destination node. So-called geographic routing algorithms have been shown to scale well in large networks thanks to their localized nature [6]. Every packet 
contains the position of its final destination, so that intermediate nodes can forward it based on their neighbors' positions relative to the destination. This only requires a local position information exchange among neighbors, and consequently has minimal overhead.

Routing plays an important role in avoiding congestion in multihop wireless networks, and therefore has a major impact on network throughput and packet delay. In dense networks, there are typically many possible paths from source to destination, and so-called multipath routing algorithms can be used to exploit path multiplicity and improve network performance, e.g., by making use of load sharing techniques. In this paper, we propose a purely localized (and therefore scalable) Geographic Load Share Routing algorithm (GLSR) that exploits path diversity to mitigate congestion in TDMA multihop wireless networks with directional antennas. While this work is motivated by the expected characteristics of the Airborne Internet, the proposed algorithm can be applied in any resource scheduling wireless network where position information is available.

\section{Network Model}

The multihop wireless network is composed of $N$ identical nodes utilizing half-duplex transceivers on the same carrier frequency (common channel). Any one particular node in the network is uniquely identified by its number $i \in\{1, \ldots, N\}$. Direct communication from node $i$ to node $j$ is represented by the directed link $(i, j)$, $i \neq j$. A link $(i, j)$ exists if a sufficiently low bit error rate (BER) can be achieved in the absence of multiple access interference. All nodes are assumed to be synchronized to a common time reference, e.g., by means of GPS. Interference is avoided by scheduling channel access in a TDMA fashion. Time is divided into repeating frames of size $T$ time slots. Transmissions start and end within slots. A TDMA schedule describes a node's transmission rights for each time slot in the frame.

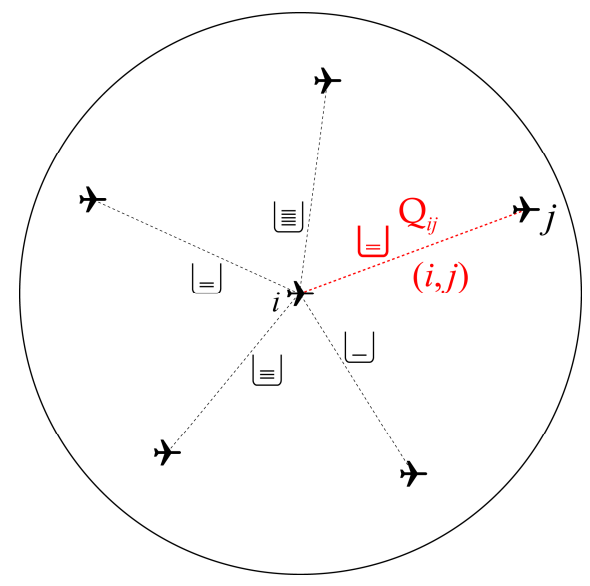

Figure 3 A node's transmission queues.

We denote by $\mathcal{N}_{i}$ the set of all one hop neighbors of node $i$. As shown in Fig. 3, every node $i$ has an outgoing link $(i, j)$ with each neighbor $j \in \mathcal{N}_{i}$, with an associated transmission queue $Q_{i j}$ where arriving packets are placed for eventual transmission over link $(i, j)$. The priority of a link $(i, j)$ is defined as

$$
p_{i j}=\lambda_{i j} / h_{i j}
$$

where $\lambda_{i j}$ is the packet arrival rate at $Q_{i j}$ (in packets/frame) and $h_{i j}$ is the number of slots currently assigned to link $(i, j)$. This is used by the link scheduling algorithm to provide fairness among competing links in the network. For each queue $Q_{i j}$ in the network, the packet arrival rate $\lambda_{i j}$ is computed at the beginning of each frame $n$ as

$$
\lambda_{i j}^{(n)}=(1-\kappa) \lambda_{i j}^{(n-1)}+\kappa \Lambda_{i j}^{(n-1)}
$$

where $\Lambda_{i j}^{(n)}$ is the number of packet arrivals at $\mathrm{Q}_{i j}$ during frame $n$. We use $\kappa=0.01$ in our simulations. The size of a packet corresponds to the duration of a time slot minus the appropriate guard interval. 


\section{Antenna and Interference Model}

Every node is equipped with a uniform circular array antenna capable of forming up to $\mathrm{K}$ beams simultaneously in arbitrary directions, as shown in Fig. 4.

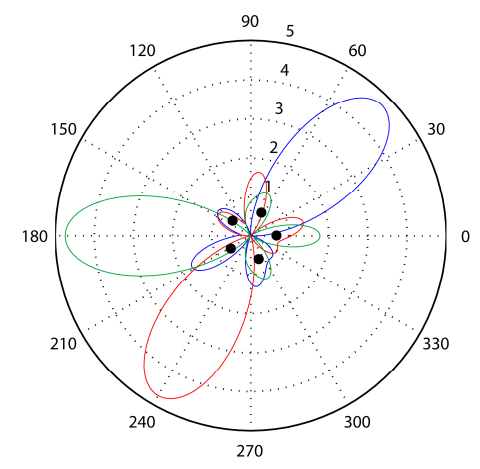

Figure 4 Multi-beam uniform circular array antenna azimuthal radiation pattern.

The antenna is assumed to be capable of fast beam switching, so that it can quickly reconfigure the directions in which it transmits or from which it receives at the beginning of every time slot. This can be achieved by using digital signal processing (DSP) technology. Beam steering is used at both ends of the link, so that the main lobe of the transmitting and receiving antenna array are pointed toward the strongest signal path, i.e., the line of sight, as shown in Fig. 5. The antenna pattern of a uniform circular array can be found in [7][8]. The half-power beamwidth, denoted by $\psi$, depends on the number of array elements.

For simplicity, all nodes are assumed to use the same transmit power. We denote the communication range by $r$ and define the maximum interference distance $\rho$ as the distance beyond which interference is assumed to be zero. For each communication link $(i, j)$, the signal-to-interference ratio (SIR) in a given slot is computed as

$$
\Gamma_{i j}=\frac{G_{i j}\left(\theta_{i j}\right) G_{j i}\left(\theta_{j i}\right) d_{i j}^{-\alpha}}{\sum_{\forall(k, l) \neq(i, j)} G_{k l}\left(\theta_{k j}\right) G_{j i}\left(\theta_{j k}\right) d_{k j}^{-\alpha} z_{k l}}
$$

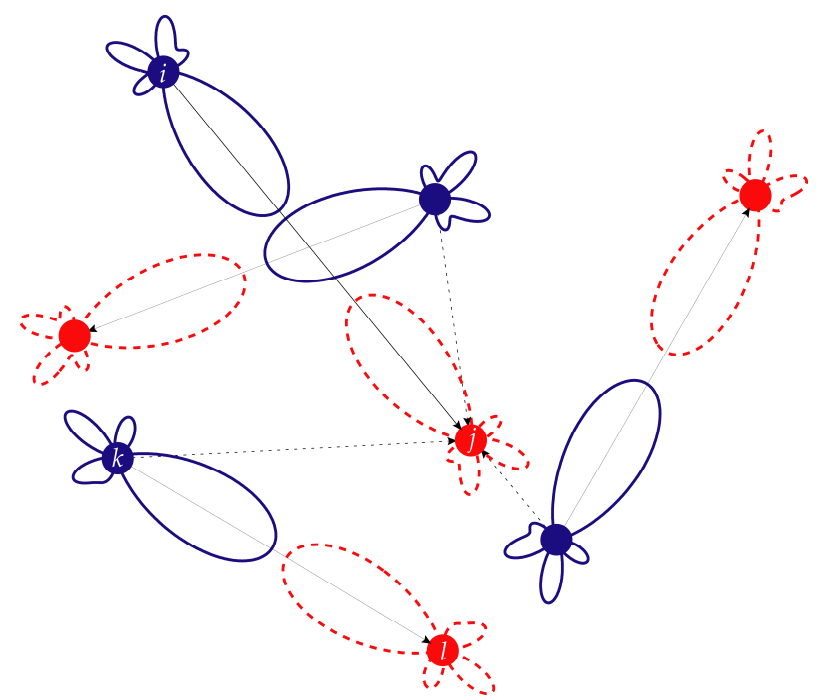

Figure 5 Example network illustrating our SIRbased interference model.

where $G_{i j}$ is the antenna radiation pattern used by node $i$ to transmit to node $j, \theta_{i j}$ is the azimuthal angle to node $j$ as seen from node $i$, $d_{i j}$ is the distance between nodes $i$ and $j, \alpha$ is the path loss exponent (we assume $\alpha=2$ in this paper), and

$z_{k l}= \begin{cases}1, & \text { if link }(k, l) \text { is scheduled and } d_{k j}<\rho \\ 0, & \text { otherwise. }\end{cases}$

Simultaneous link activation in a given time slot is limited by the following constraints:

$\left(\mathbf{c}_{1}\right)$ Half duplex operation: A node cannot transmit and receive simultaneously.

$\left(\mathbf{c}_{2}\right)$ A node may activate at most $\mathrm{K}$ outgoing (transmit mode) or incoming (receive mode) links simultaneously.

$\left(c_{3}\right)$ The signal-to-interference ratio at all scheduled receivers must be above a specified communication threshold $\gamma_{\mathrm{o}}$.

We assume that link $(i, j)$ can communicate a packet without error if $\Gamma_{i j}>\gamma_{\mathrm{o}}$. 


\section{Link Scheduling}

We use the distributed Spatial TDMA (STDMA) link scheduling algorithm proposed by Grönkvist [9]. In this section, we provide a brief summary of the essential aspects of the algorithm. For a detailed description, see [9]. The distributed STDMA algorithm consists of the following steps:

1. Nodes that have entered the network exchange local information with their neighbors.

2 . The link with highest priority in its local neighborhood assigns itself a time slot.

3. The local schedule is then updated within the local neighborhood of the link, and a new link has highest priority. This process (2.-3.) is continued until there are no available slots.

The local neighborhood $\mathcal{L}_{i j}$ of link $(i, j)$ is defined as the set of all other links $(k, l)$ in the network whose transmitter $k$ is within interference distance of $j$ and/or whose receiver $l$ is within interference distance of $i$, i.e.,

$$
\mathcal{L}_{i j}=\left\{(k, l): d_{k j}<\rho\right\} \cup\left\{(k, l): d_{i l}<\rho\right\} .
$$

The STDMA algorithm is run in parallel for each link, i.e., each link can be seen as a separate process. As shown in Fig. 6, a link process can be in one of three states: Active (A), Waiting (W), or aSleep (S). Transitions between states are triggered by the following conditions:

$$
\begin{aligned}
\mathrm{P}_{0} \equiv & \text { a neighbor link has higher priority, i.e., } \\
& \exists(k, l) \in \mathcal{L}_{i j} \text { s.t. } p_{i j}<p_{k l} \\
\mathrm{P}_{1} \equiv & \text { no neighbor link has higher priority, i.e., } \\
& p_{i j}>p_{k l}, \forall(k, l) \in \mathcal{L}_{i j} \\
\mathrm{~A}_{0} \equiv & \text { there are no available slots for link }(i, j) \\
\mathrm{A}_{1} \equiv & \text { there are available slots for link }(i, j) \\
\mathrm{A}_{2} \equiv & p_{i j} \text { is lower than the average priority in } \\
& \text { the neighborhood, i.e., } p_{i j}<\frac{\sum_{\mathcal{L}_{i j}} \lambda_{k l}}{\sum_{\mathcal{L}_{i j}} h_{k l}}
\end{aligned}
$$

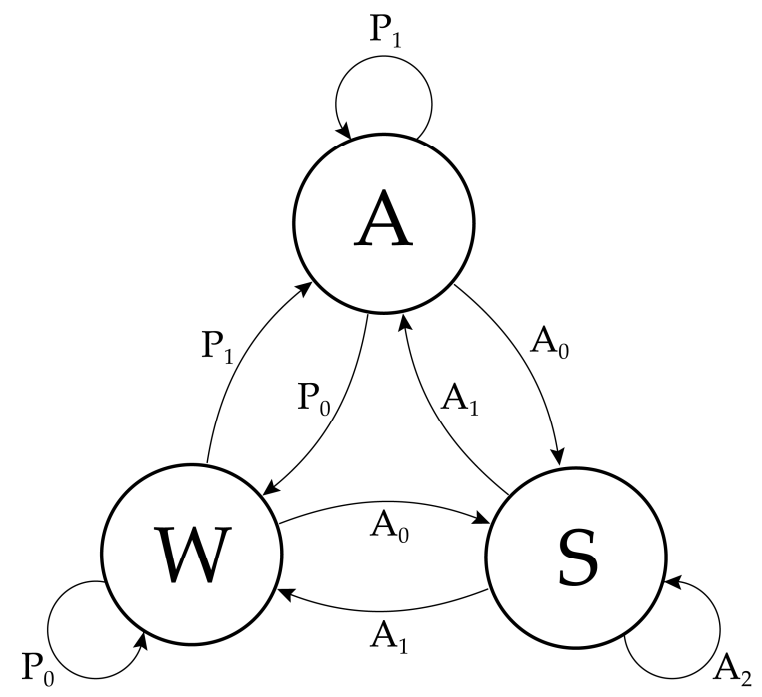

Figure 6 Link states in STDMA scheduling algorithm.

where $p_{i j}$ is given by (1) and slot availability is defined by constraints $\left(\mathbf{c}_{1}\right)-\left(\mathbf{c}_{3}\right)$ above. In this way, link priority decides in which order links may attempt to assign themselves a time slot. Asleep links are not considered in terms of priority and do not participate in the contention for resources.

In order to spread its slot allocations evenly over the frame, an active link will choose the furthest available slot from its current allocations. This is advantageous in terms of packet delay [10]. If no slot is available, the link will enter the aSleep (S) state, unless it can steal an allocation from a lower priority link in the local neighborhood. A link $(i, j)$ is only permitted to steal a time slot from another link $(k, l)$ if the priority of the stealing link is greater than the other link's priority after the loss of a time slot, i.e.,

$$
\frac{\lambda_{i j}}{h_{i j}}>\frac{\lambda_{k l}}{h_{k l}-1} .
$$

A time slot assignment is maintained for as long as possible until either it can no longer be used reliably or it is stolen by a higher priority link. 
Node movement will cause topological changes and modify the interference geometry, so that allocations that were compatible at one time cease to be so at a later time. This leads to rescheduling. However, the topology change rate in the Airborne Internet is expected to be low, given the scale (communication range vs flight speed) and the fact that aircraft fly in a kind of formation. ${ }^{1}$ Therefore, we will consider static topologies in our simulations.

We assume the existence of a separate omnidirectional control channel (OCC) to exchange interference and scheduling information among neighbors. Exactly how the OCC is implemented is out of the scope of this paper. However, aircraft already use beaconing mechanisms such as ADS-B (Automatic Dependent Surveillance Broadcast) to periodically broadcast their state vector, including position, speed, etc., and we suggest that such systems could be extended to include the above functionality.

\section{Geographic Load Share Routing}

Previous work on routing in aeronautical ad hoc networks has focused on exploiting geolocalization information [12]-[15]. In this section we describe our main contribution, the Geographic Load Share Routing (GLSR) algorithm. GLSR extends the well-known Greedy Perimeter Stateless Routing (GPSR) algorithm [16] to exploit the multiplicity of source-destination paths in moderate and high density multihop wireless networks. Greedy routing has inspired many extensions in the context of sensor networks, where energy consumption is an important

\footnotetext{
${ }^{1}$ As a result of passenger demand, time zone differences and airport noise restrictions, much of the North Atlantic air traffic contributes to two major alternating flows: a westbound flow departing Europe in the morning, and an eastbound flow departing North America in the evening. The effect of these flows is to concentrate most of the traffic unidirectionally [11].
}

performance factor [17], as well as in vehicular networks with carrier-sense multiple access schemes [18]. However, these extensions do not appear relevant to the Airborne Internet. We will therefore use GPSR as a benchmark in our simulations.

In the Airborne Internet, the distance between two nodes $i$ and $j$ is defined as the great circle angular distance $\delta_{i j}$, given (in radians) by

$$
\delta_{i j}=\cos ^{-1}\left(\sin \theta_{i} \sin \theta_{j}+\cos \theta_{i} \cos \theta_{j} \cos \left(\varphi_{i}-\varphi_{j}\right)\right)
$$

where $\left(\theta_{k}, \varphi_{k}\right)$ denote the latitude and longitude of node $k$, respectively.

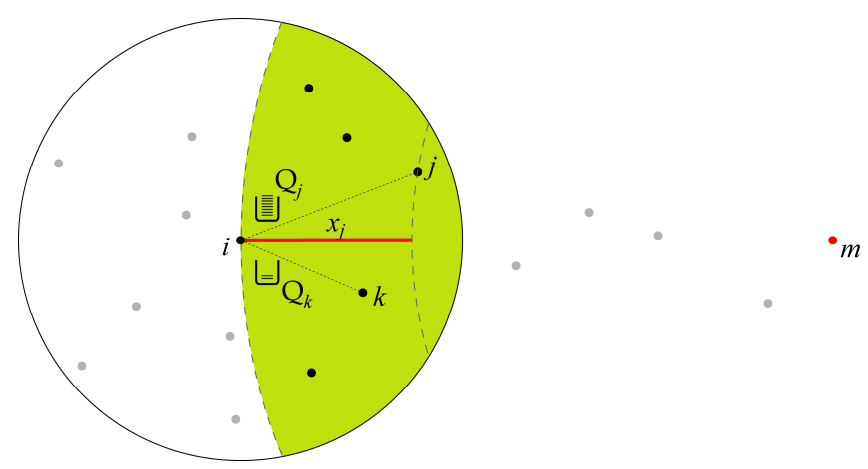

\section{Figure 7 Geographic Load Share Routing (GLSR).}

Consider a packet arriving at node $i$ with destination $m$, as shown in Fig. 7. The packet's advance toward $m$ if forwarded to neighbor $k$, denoted by $x_{k}$, is defined as the difference between $i$ 's and $k$ 's geographical distance to $m$, i.e.,

$$
x_{k}=\delta_{i m}-\delta_{k m} .
$$

In GPSR, packets are forwarded to the neighbor that is geographically closest to their destination, i.e., that yields the greatest advance. In a link scheduling context, such as STDMA, a node $i$ buffers arriving packets in a separate transmission queue $Q_{j}$ (we drop the $i$ subscript in the sequel) for each outgoing link $(i, j)$. Thus, 
GPSR places a packet arriving at node $i$ with destination $m$ in $\mathrm{Q}_{j}$ such that

$$
x_{j}=\max _{k \in \mathcal{N}_{i}}\left\{x_{k}\right\}, x_{k}>0 .
$$

If the packet arrival rate at $Q_{j}$ is higher than the number of slots assigned to link $(i, j)$, i.e., $\lambda_{i j}>h_{i j}$, the queue will grow in size, since packets arrive at a greater rate than they are transmitted (see Fig. 7). This will lead to increased queueing delay of packets, and may eventually result in packets being dropped, unless link $(i, j)$ is able to obtain additional slots.

In order to reduce the probability of link congestion, we introduce GLSR. We define a packet's speed of advance toward destination $m$ for neighbor $k$ as

$$
v_{k}=\frac{x_{k}}{\widehat{\mathrm{Q}}_{k}+1}
$$

where $\hat{Q}_{k}$ is the average number of packets in $\mathrm{Q}_{k}$, measured over a given time window. GLSR places a packet arriving at node $i$ with destination $m$ in $Q_{j}$ such that

$$
v_{j}=\max _{k \in \mathcal{N}_{i}}\left\{v_{k}\right\}, v_{k}>0 .
$$

If the destination $m$ is a neighbor of node $i$, the packet is simply placed in $\mathrm{Q}_{m}$. The average queue size $\widehat{Q}_{k}$ is computed at the beginning of each frame $n$ as

$$
\widehat{\mathrm{Q}}_{k}^{(n)}=(1-\beta) \widehat{\mathrm{Q}}_{k}^{(n-1)}+\beta \overline{\mathrm{Q}}_{k}^{(n-1)}
$$

where $\overline{\mathrm{Q}}_{k}^{(n)}$ is the queue size upon arrival, averaged for all packet arrivals in frame $n$. We use $\beta=0.01$ in our simulations.

Intuitively, the idea behind GLSR is to choose the neighbor which simultaneously maximizes the packet's advance toward destination, as in GPSR, and minimizes the packet's expected queueing delay, which is proportional to the average queue size by Little's formula [19]. However, these two objectives cannot in general be fulfilled simultaneously. Thus, the solution adopted by GLSR is to maximize the ratio between advance and queueing delay, represented by the speed of advance metric. In this way, GLSR performs load sharing among all neighbors with positive advance (shaded area in Fig. 7), choosing at any time the next hop with the highest speed of advance toward the destination.

Note that we use the average queue size, rather than the instantaneous value, as in a Join the Shortest Queue (JSQ) discipline. In a traffic sensitive STDMA network, as described in this paper, the link capacities (i.e., the service rates of the transmission buffers) are dynamically adapted according to the link demands. In order to give the scheduling enough time to react to an increase in demand before starting to detour packets, we need to attenuate the short-term fluctuations in buffer size. Note also that information about the queue size is local to the forwarding node and does not need to be sent over the channel, thus introducing no additional overhead.

\section{Simulation Results}

We have conducted simulations using the OMNeT++ discrete event network simulator along with the INET framework [20]. As shown in Fig. 8, we distribute $N=100$ aircraft nodes uniformly over a rectangular area of size 800 nmi x $400 \mathrm{nmi}$, according to a two dimensional Poisson point process. This area is roughly half the size of the North Atlantic gap (Fig. 1). The chosen aircraft density corresponds approximately to the peak instantaneous aircraft count in this region [21]. In addition, a ground station node is placed on one side of the rectangle, and acts as an Internet Gateway (IGW). 


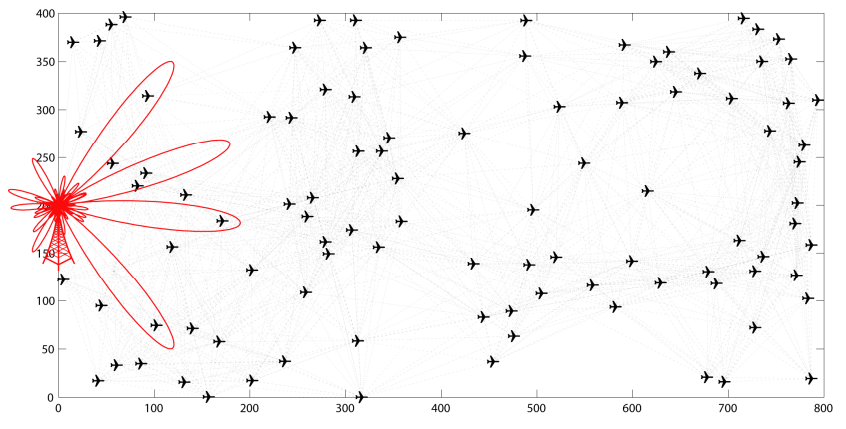

Figure 8 Simulation scenario.

Table 1. Simulation Settings

\begin{tabular}{|l|l|}
\hline Parameter & Value \\
\hline$N$ & 100 nodes \\
\hline$r$ & $200 \mathrm{nmi}$ \\
\hline$\rho_{\mathrm{A} 2 \mathrm{G}}$ & $225 \mathrm{nmi}$ \\
\hline$\rho_{\mathrm{A} 2 \mathrm{~A}}$ & $450 \mathrm{nmi}$ \\
\hline$\gamma_{\mathrm{o}}$ & $6 \mathrm{~dB}$ \\
\hline$\psi$ & $10^{\circ}$ \\
\hline $\mathrm{K}_{\mathrm{IGW}}$ & 8 beams \\
\hline $\mathrm{K}_{\mathrm{AC}}$ & 1 beam \\
\hline$T$ & 100 slots \\
\hline
\end{tabular}

We assume multibeam capability only at the $\mathrm{IGW}$, with $\mathrm{K}_{\mathrm{IGW}}=8$ and $\mathrm{K}_{\mathrm{AC}}=1$. Other than this, the IGW behaves just as any other node. The radio communication range, both air-to-ground and air-to-air, is set at $r=200 \mathrm{nmi}$, whereas the maximum interference distance is $\rho_{\mathrm{A} 2 \mathrm{G}}=225$ nmi for air-to-ground and $\rho_{\mathrm{A} 2 \mathrm{~A}}=450 \mathrm{nmi}$ for airto-air, corresponding to the aircraft's line-ofsight horizon in each case, for a typical flight level of $35000 \mathrm{ft}$. Note that in a real environment, as shown in Fig. 1, there may be more than one IGW available at a given time. However, there will be times when connectivity is restricted to one single IGW. Such critical situations most clearly highlight the ability of GLSR to mitigate link congestion.
Internet downstream traffic is generated at the IGW based on a Poisson traffic model with mean value $\lambda$ packets/slot. Each new packet has a randomly chosen destination among the $N$ aircraft nodes, so that, on average, every aircraft receives $\lambda / N$ packets/slot. In addition, upstream traffic (from the aircraft to the IGW) is generated at each aircraft based on a Poisson traffic model with mean value $0.1 \lambda / N$ packets/slot. Thus, the downstream/upstream asymmetry factor is 1:10. Each simulation run corresponds to a random network topology and has a duration of 1000 frames, long enough to collect the metrics of interest. Queues have a buffer size of 20 packets. Packets arriving at a full queue are dropped.

Fig. 9 shows the network throughput, packet delivery ratio (PDR) and average end-toend delay, obtained with the simulation settings given in Table 1. In each case, the curves show the average over 100 random topologies. As can be seen, buffer overflow is reached by GLSR at significantly higher throughput levels. This is due to GLSR's ability to perform load sharing among all available neighbors in the direction of the destination, spatially spreading traffic based on queue dynamics.

We define the maximum throughput $\lambda^{*}$ that can be sustained by the network as the value of $\lambda$ for which $95 \%$ of the generated packets are successfully delivered, i.e., $\operatorname{PDR}\left(\lambda^{*}\right)$ $=95 \%$. The value of $\lambda^{*}$ depends on the network topology. Fig. 10 shows the histograms of the maximum throughput for 1000 random topologies, for $\gamma_{\mathrm{o}}=6 \mathrm{~dB}$ and $\gamma_{\mathrm{o}}=9 \mathrm{~dB}$. As can be seen in the histograms, the ability of GLSR to avoid congestion by detouring packets to alternative links is constrained by the degree of spatial reuse in the network. 


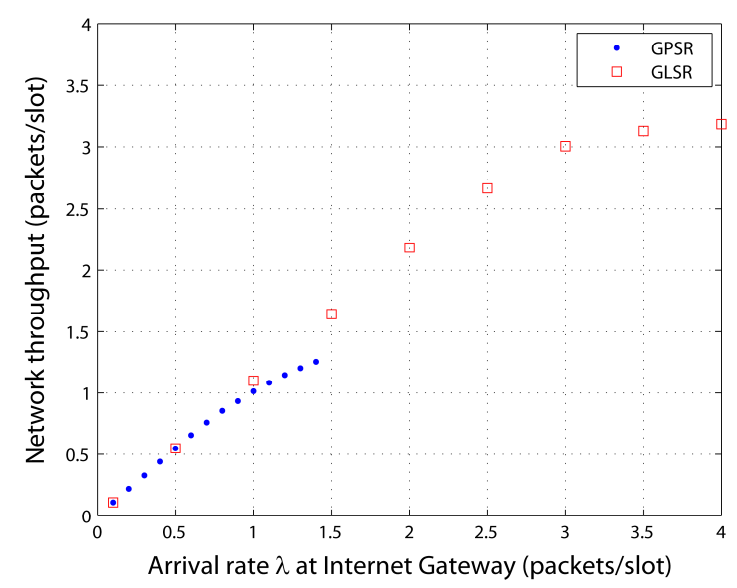

(a) Network throughput

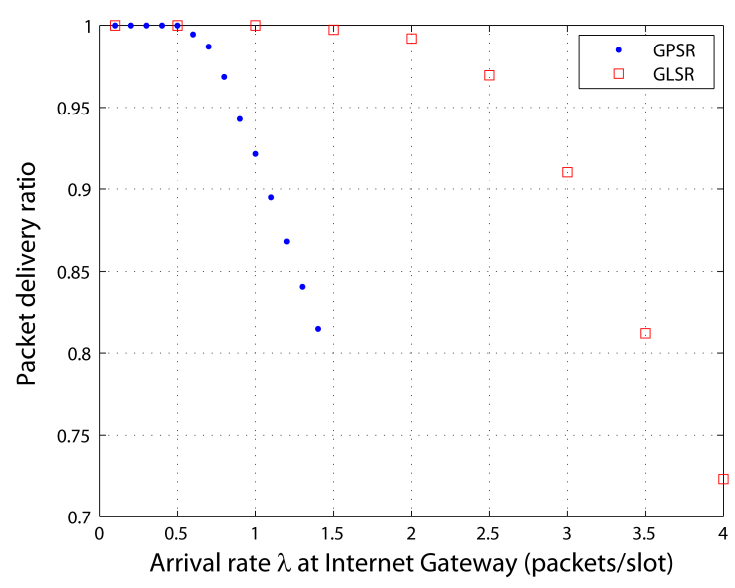

(b) Packet delivery ratio

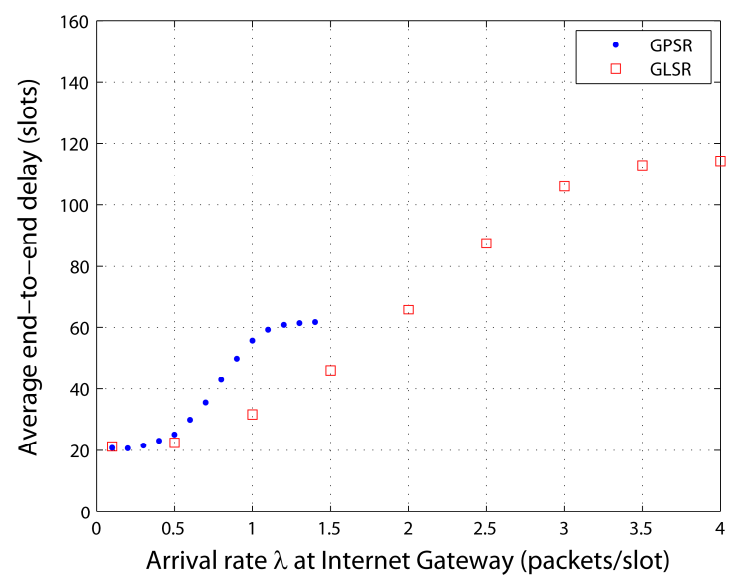

(c) Average end-to-end delay

Figure 9 Simulation results for the settings given in Table 1.

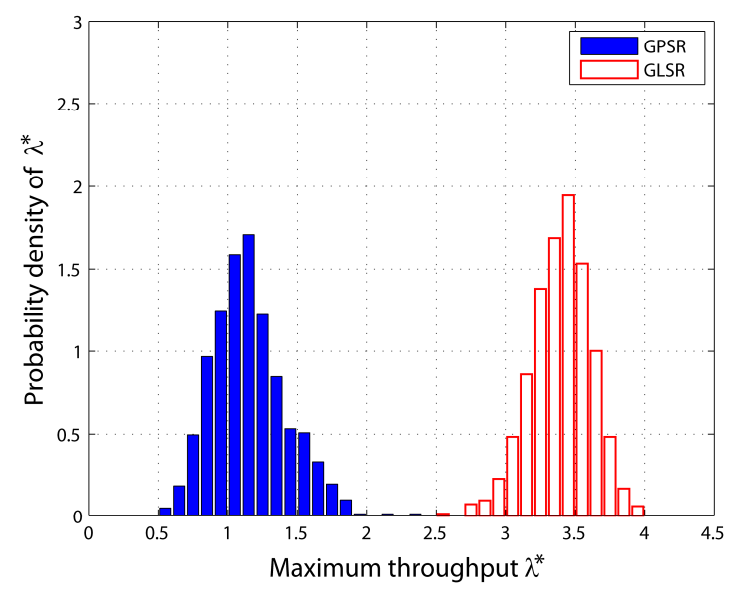

(a) $\gamma_{\mathrm{o}}=6 \mathrm{~dB}$

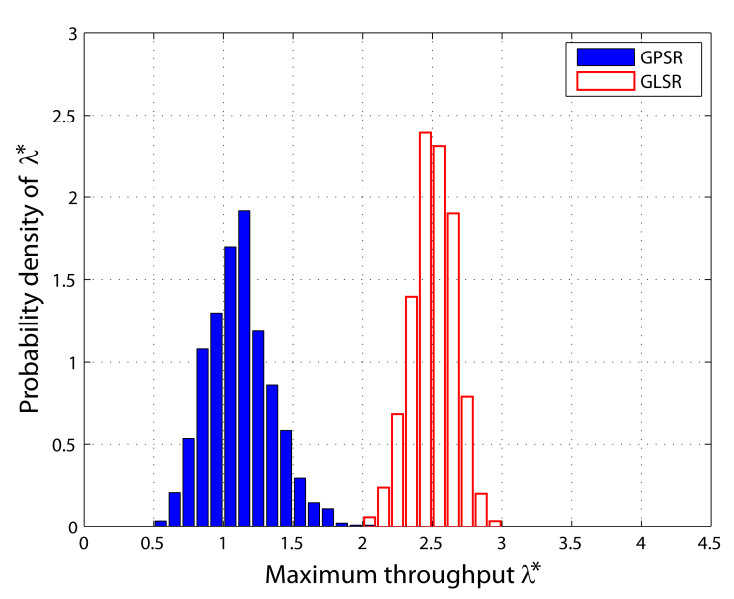

(b) $\gamma_{\mathrm{o}}=9 \mathrm{~dB}$

\section{Figure 10 Histograms of the maximum throuhput $\lambda^{*}$.}

As we increase the communication threshold $\gamma_{\mathrm{o}}$ from $6 \mathrm{~dB}$ to $9 \mathrm{~dB}$, we reduce the spatial reuse in the network, i.e., fewer links can transmit simultaneously in a given time slot. This means there is less available capacity for alternative links to help avoid congestion of overloaded links. With $\gamma_{\mathrm{o}}=6 \mathrm{~dB}$, GLSR achieves on average 3 times as much throughput as GPSR.

Note that our antenna model is relatively pessimistic for an aeronautical environment. Aircraft are likely to be equipped with advanced 
smart antenna technology capable of null steering, thus reducing the impact of side lobes. Moreover, we have used the maximum possible interference distance $\rho$, given by the horizon. In reality, the interference region of a transmission may be reduced by different environmental factors, such as clouds, rain, etc., depending on the frequency spectrum used, thus increasing the spatial reuse that can be achieved in the network.

Fig. 11 illustrates the link activity resulting from GPSR and GLSR routing (for a downstream only scenario). By virtue of its flexibility, GLSR exploits the diversity of paths available from source to destination, significantly increasing the maximum network throughput achievable.
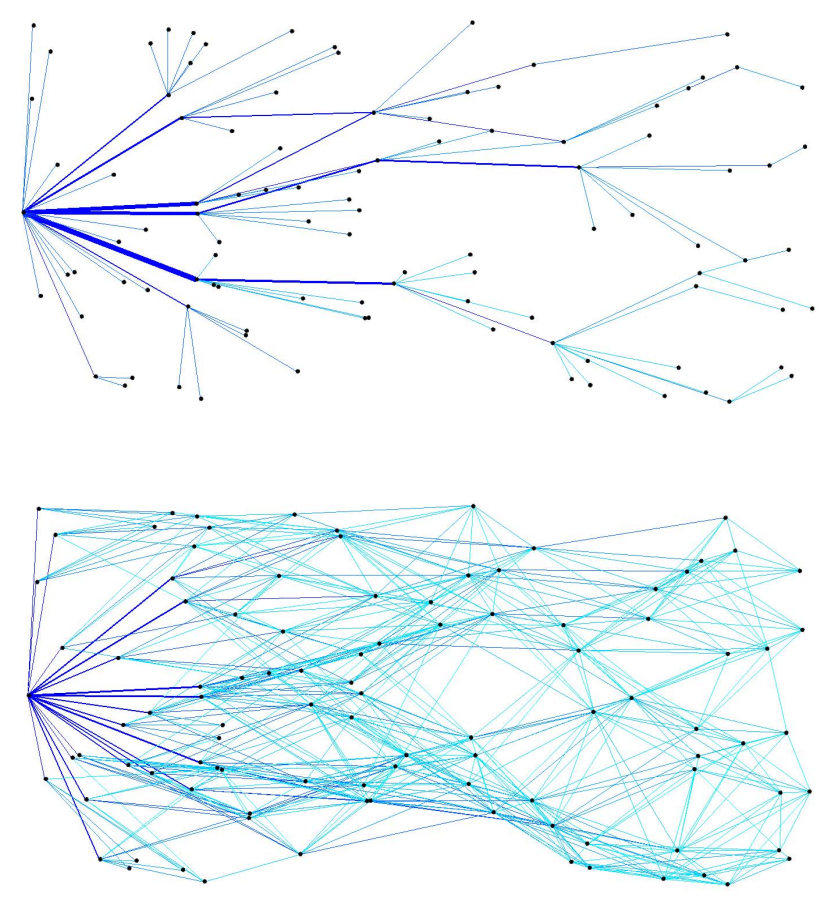

Figure 11 Comparison of GPSR and GLSR active links in a downstream only scenario. (Link width represents packet arrival rate at corresponding transmission queue.)

\section{Conclusion}

The research presented in this paper is motivated by the unique characteristics of the Airborne Internet, an envisioned large scale wireless mesh network formed by civil aviation aircraft networked with each other via highly directional air-to-air radio links. The focus of our contribution is on avoiding link congestion in multihop wireless networks via adaptive geographic routing of packets. As an analogy, road congestion in a large city can be mitigated by controlling street (link) capacity, e.g., changing the number of lanes (slots) in a given direction, but much more so by rerouting cars away from roads that are close to saturation. Geographic Load Share Routing (GLSR) combines position-based packet forwarding with a Join the Shortest Queue (JSQ) principle by defining a new metric, the speed of advance of a packet. By locally maximizing this metric, every router spreads incoming traffic among all nodes in the direction of the destination, avoiding excessive queueing delay at its transmission buffers. The result is an increase in the maximum throughput that can be sustained by the network, without packets being unnecessarily dropped when they can be successfully rerouted. The ability to exploit this flexibility depends on the spatial reuse of the underlying network. For the simulated scenario, an increase in network throughput of $200 \%$ on average has been shown.

\section{References}

[1] DirecNet Task Force Open Session, San Diego, Feb 2007, https://www.direcnet.us/direcnet

[2] Airborne Internet Consortium (AIC), http://www.airborneinternet.org

[3] W. McNary, Transformational Aircraft Communication Using a Broadband Mesh Network, $7^{\text {th }}$ ICNS Conference, May 2007. 
[4] S. Yi and Y. Pei, On the Capacity Improvement of Ad Hoc Wireless Networks using Directional Antennas, ACM MobiHoc 2003, Annapolis, MD, June 2003.

[5] R. Ramanathan, J. Redi, C. Santiváñez, D. Wiggins and S. Polit, Ad Hoc Networking with Directional Antennas: A Complete System Solution, IEEE Journal on Selected Areas in Communications, March 2005, Volume 23, Issue 3, pp. 496-506.

[6] M. Mauve, J. Widmer and H. Hartenstein, A Survey on Position-based Routing in Mobile Ad Hoc Networks, IEEE Network, November 2001, Volume 15, Issue 6, pp. 30-39.

[7] C. A. Balanis, Antenna Theory: Analysis and Design, 3rd edition, Wiley-Interscience, April 2005.

[8] C. Moser, Ad Hoc Networking with Beamforming Antennas: Modeling, Visualization and Connectivity, Diploma Thesis, Technical University of Munich (TUM), Munich, Germany, December 2004.

[9] J. Grönkvist, Interference-based Scheduling in Spatial Reuse TDMA, Ph.D. Thesis, Royal Institute of Technology (KTH), Stockholm, Sweden, 2005.

[10] R. Nelson and L. Kleinrock, Spatial TDMA: A Collision-Free Multihop Channel Access Protocol, IEEE Transactions on Communications, September 1985, Volume 33, Issue 9, pp. 934-944.

[11] North Atlantic Minimum Navigation Performance Specifications (MNPS) Airspace Operations Manual, Edition 2008, published on behalf of the North Atlantic Systems Planning Group (NAT SPG) by the European and North Atlantic Office of ICAO, 2008.

[12] E. Sakhaee and A. Jamalipour, The Global InFlight Internet, IEEE Journal on Selected Areas in Communications, September 2006.
[13] E. Sakhaee, A. Jamalipour and N. Kato, Aeronautical Ad Hoc Networks, IEEE WCNC 2006.

[14] M. Iordanakis, D. Yannis, K. Karras, G. Bogdos, G. Dilintas, M. Amirfeiz, G. Colangelo and S. Baiotti, Ad-hoc Routing Protocol for Aeronautical Mobile Ad-Hoc Networks, 5th Int. Symposium on Communication Systems, Networks and Digital Signal Processing (CSNDSP), July 2006.

[15] H. D. Tu and S. Shimamoto, Mobile Ad-Hoc Network Based Relaying Data System for Oceanic Flight Routes in Aeronautical Communications, International Journal of Computer Networks and Communications (IJCNC), Volume 1, Issue 1, April 2009.

[16] B. Karp and H. T. Kung, Greedy Perimeter Stateless Routing for Wireless Networks, in Proceedings of the Sixth Annual ACM/IEEE International Conference on Mobile Computing and Networking (MobiCom 2000), Boston, MA, pp. 243-254, August 2000.

[17] T. Melodia, D. Pompili and I. F. Akyildiz, On the Interdependence of Distributed Topology Control and Geographical Routing in Ad Hoc and Sensor Networks, IEEE Journal of Selected Areas in Communications (JSAC), Volume 23, Issue 3, pp. 520-532, March 2005.

[18] A. Festag, R. Baldessari and H. Wang, On Power-Aware Greedy Forwarding in Highway Scenarios, Proceedings of 5th International Workshop on Intelligent Transportation (WIT07), March 2007.

[19] M. Zukerman, Introduction to Queueing Theory and Stochastic Teletraffic Models, 2010.

[20] OMNeT++, http://www.omnetpp.org

[21] D. Medina, F. Hoffmann, S. Ayaz and C.-H. Rokitansky, Feasibility of an Aeronautical Mobile Ad Hoc Network Over the North Atlantic Corridor, IEEE SECON 2008, San Francisco, CA, June 2008. 


\section{Email Addresses}

Daniel Medina: Daniel.Medina@dlr.de

Felix Hoffmann: Felix.Hoffmann@dlr.de

Francesco Rossetto: Francesco.Rossetto@dlr.de

Carl-Herbert Rokitansky: roki@cosy.sbg.ac.at
2010 Integrated Communications Navigation and Surveillance (ICNS) Conference

May 11-13, 2010 